\title{
MODELO BIOECOLÓGICO DE URIE BRONFENBRENNER E INSERÇÃO ECOLÓGICA: UMA METODOLOGIA PARA INVESTIGAR FAMÍLIAS RURAIS ${ }^{1}$
}

\author{
Juliana Graciela Vestena Zillmer², Eda Schwartz³, Rosani Manfrin Muniz', Sonia Maria Könzgen Meincke
}

${ }^{1}$ Trabalho extraído da dissertação - Práticas de cuidado no contexto das famílias rurais à pessoa com câncer, apresentada à Faculdade de Enfermagem da Universidade Federal de Pelotas, 2009.

${ }^{2}$ Doutoranda do Programa de Pós-Graduação em Enfermagem da Universidade Federal de Santa Catarina. Bolsista CAPES. Rio Grande do Sul, Brasil. E-mail: juzillmer@gmail.com

${ }^{3}$ Doutora em Enfermagem. Docente da Faculdade de Enfermagem e Obstetrícia da Universidade Federal de Pelotas (UFPeL). Rio Grande do Sul, Brasil. E-mail: eschwartz@terra.com

${ }^{4}$ Doutora em Enfermagem. Docente da Faculdade de Enfermagem e Obstetrícia da UFPeL. Rio Grande do Sul, Brasil. E-mail: romaniz@terra.com

${ }^{5}$ Doutora em Enfermagem. Docente da Faculdade de Enfermagem e Obstetrícia da UFPeL. Rio Grande do Sul, Brasil. E-mail: meincke@terra.com

RESUMO: Este trabalho tem o objetivo de relatar a experiência na utilização da metodologia de Inserção Ecológica e o Modelo Bioecológico do Desenvolvimento Humano, de Urie Bronfenbrenner, e sua aplicação em pesquisa com famílias rurais que vivenciam o câncer. A inserção ecológica envolveu o acompanhamento de três famílias da área rural, que tinham um de seus membros em tratamento quimioterápico no Serviço de Oncologia do Hospital-Escola da Universidade Federal de Pelotas, Rio Grande do Sul, Brasil. A utilização do Modelo e da Inserção permitiu a compreensão dos processos que envolvem a família rural no cuidar da pessoa com câncer. Entendemos que a enfermagem, por ser uma profissão comprometida com o cuidar, além do biológico, ao analisar os fenômenos por meio dos quatro núcleos do modelo (pessoa, processo, contexto e tempo), promoverá ações de cuidado voltadas para a singularidade de cada família.

DESCRITORES: População rural. Pesquisa qualitativa. Famílias. Câncer.

\section{URIE BRONFENBRENNER'S BIOECOLOGICAL MODEL AND ECOLOGICAL INSERTION: A METHODOLOGY TO INVESTIGATE RURAL FAMILIES}

\begin{abstract}
The following paper aims to report the experience in using the Ecological Insertion Methodology and Urie Bronfenbrenner's Human Development Bioecological Model and their use in research involving rural families experiencing cancer. Ecological insertion involved the follow-up of three rural families, who had one of their family members undergoing chemotherapy treatment in the Oncology Services at the Federal University of Pelotas Hospital, Rio Grande do Sul, Brazil. The use of the Model and the Insertion allowed to understand the processes involving a rural family while caring for a person with cancer. It is understood that nursing, being a profession committed to care beyond biological, analyzing the phenomena through the four axes of the model (person, process, context, and time) will promote care actions geared towards the singularity of each family.
\end{abstract}

DESCRIPTORS: Rural population. Qualitative research. Families. Cancer.

\section{MODELO BIOECOLÓGICO DE URIE BRONFENBRENNER E INSERCIÓN ECOLÓGICA: UNA METODOLOGÍA PARA INVESTIGAR LAS FAMILIAS RURALES}

RESUMEN: Este trabajo tiene como objetivo relatar la experiencia en la utilización de la metodología de Inserción Ecológica y el Modelo Bioecológico del Desarrollo Humano de Urie Bronfenbrenner y su aplicación en la investigación con familias que habitan el medio rural y experimentan el cáncer. La inserción ecológica involucró el monitoreo de tres familias del área rural que tenían uno de sus miembros en tratamiento de quimioterapia en el Servicio Oncológico del Hospital Escuela de la Universidad Federal de Pelotas, Rio Grande do Sul, Brasil. La utilización del Modelo y de la Inserción posibilitó la comprensión de los procesos que involucran la familia rural en el acto de cuidar personas con cáncer. Entendemos que la enfermería, por ser una profesión comprometida con el cuidado que va más allá de lo biológico, cuando analiza los fenómenos a través de los cuatro núcleos del modelo (persona, proceso, contexto y tiempo) impulsará acciones de cuidado centradas en la singularidad de cada familia.

DESCRIPTORES: Población rural. Pesquisa cualitativa. Familias. Cáncer. 


\section{INTRODUÇÃO}

A complexidade do contexto social vem exigindo das pessoas o desenvolvimento de pesquisas que se utilizem de metodologias que possibilitem investigar famílias em situações de adoecimento e vulnerabilidade e, se for necessário, elaborar estratégias para intervir na sua realidade. A família, como foco central na pesquisa, está cercada por avanços teóricos e metodológicos, porém ainda insuficientes, se considerarmos sua complexidade e multidimensionalidade. ${ }^{1}$

As "estruturas teóricas sobre a família, provenientes dos conhecimentos construídos nas disciplinas, especialmente nas Ciências Sociais e na Psicologia, têm proporcionado embasamento para guiar as pesquisas na área da Enfermagem". 1:24 Entre esses constructos, um dos referenciais sistêmicos utilizados é o Modelo Bioecológico de Urie Bronfenbrenner. ${ }^{2-5}$ Assim, ao construir conhecimento a partir de pesquisas, tenta-se encontrar novas maneiras de olhar e compreender a família no seu ambiente, e de buscar caminhos que possibilitem uma direção para a prática do cuidado de enfermagem, no contexto e cotidiano das famílias.

O Modelo Bioecológico possibilita a investigação e a análise do fenômeno por meio de quatro núcleos inter-relacionados: pessoa, processo, contexto e tempo; e também é denominado de modelo PPCT. Ele promove o conhecimento de como essas pessoas ou grupo familiar vivenciam determinada experiência (processo), inseridas em diferentes contextos. Esses contextos possuem aspectos físicos, sociais e valorativos, que os caracterizam e exercem influência na pessoa e no grupo familiar, pela continuidade (tempo) e estabilidade do sistema de relações e da construção de suas rotinas, de sua biografia e história social. ${ }^{6}$

Entende-se que a aplicação de um referencial sistêmico, em investigações de enfermagem que buscam compreender famílias, é de extrema relevância, pois várias pesquisas nessa área envolvem grupos singulares, com diferentes contextos culturais, sociais e econômicos, vivenciando situações diversas para serem analisadas. Desse modo, é importante que pesquisadores interessados por essa abordagem aprofundem seus conhecimentos e assim obtenham subsídios que facilitem a sua utilização em pesquisas.

O objetivo deste artigo é o de relatar a experiência das autoras no uso dessa metodologia e referencial, em que se buscou conhecer as práticas de cuidado das famílias rurais que vivenciam o câncer. Este trabalho discorre sobre a importância do método, principais conceitos e características, e a sua aplicação para a enfermagem.

\section{APLICAÇÃO DA METODOLOGIA}

As práticas de cuidado, no contexto das famílias rurais, foram analisadas pela inserção nos ambientes em que a pessoa com câncer e sua família participavam, como o Serviço de Oncologia, o domicílio, e a comunidade. Além disso, utilizou-se de relatos da pessoa e sua família sobre o contexto em que viviam, uma vez que é necessário examinar o ambiente, conforme ele é percebido e experienciado pelos indivíduos. ${ }^{7}$

O estudo foi norteado pelo referencial teórico do Modelo Bioecológico de Urie Bronfenbrenner. ${ }^{8} \mathrm{Na}$ busca de ferramentas metodológicas que fizessem uma aproximação com as famílias rurais, o método escolhido foi o da inserção ecológica, que tem como objetivo avaliar os processos de interação das pessoas com o contexto no qual estão se desenvolvendo. ${ }^{9}$ Foi realizado com três famílias da área rural que, no período da coleta, tinham um de seus membros em tratamento quimioterápico, no Serviço de Oncologia do Hospital-Escola da Universidade Federal de Pelotas, na Região Sul do Brasil.

A preferência pelo Modelo Bioecológico se deve à possibilidade que apresenta de compreender o desenvolvimento humano como um processo contínuo, que depende das interações vivenciadas pelas pessoas, a partir das interligações que elas criam com seu contexto e sua história de vida. Dessa forma, as experiências vivenciadas pelas famílias rurais se mostram como um forte determinante nas possibilidades de realização de práticas de cuidado. Esse modelo possibilitou examinar a realização de práticas de cuidado pelas famílias, no cuidar a pessoa com câncer, levando em conta os múltiplos fatores que atuam para a produção do cuidado, incluindo desde as interações vivenciadas no microssistema familiar, até o macrossistema, proporcionando aos profissionais de saúde uma visão sistêmica da situação.

A partir da interação com o contexto da família rural, foi possível conhecer quais eram as práticas de cuidado desenvolvidas por essas famílias no cuidado à pessoa com câncer. Tanto o paciente como sua família passavam por um 
processo perturbador, com intenso sofrimento e sensação de morte iminente. Sentimentos de raiva e revolta também foram observados no momento de coleta de dados, assim como ocasiões em que a família compartilhava o sofrimento e buscava forças para superá-lo. Pode-se, então, dizer que o contexto tem, portanto, papel-chave, já que é nele que as interações e os processos proximais acontecem. ${ }^{6} \mathrm{O}$ processo proximal surge pela interação recíproca e complexa de pesquisadores, participantes, objetos e símbolos presentes no contexto imediato, constituindo a base de toda investigação que adota a Inserção Ecológica. ${ }^{9}$ Esta apoia-se em cinco aspectos indispensáveis para o estabelecimento de processos proximais:

(a) Pesquisadores e participantes interagem e se engajam em uma tarefa comum - Neste aspecto, procuramos nos engajar em atividades com as famílias, as quais compreenderam os momentos da realização das refeições, da construção do genograma e ecomapa, com a participação de todas as pessoas da família; e o mesmo se deu durante as entrevistas, nas orientações sobre o tratamento, na visualização de eventos da família e da pessoa com câncer e a família.

(b) Existe a necessidade diversos encontros, ao longo de um considerável período de tempo Neste aspecto, realizamos 10 encontros com cada uma das famílias. Destes, cinco foram no Serviço de Oncologia e cinco no domicílio, sendo desenvolvidos em um período de seis meses. Tiveram início com uma etapa de vinculação caracterizada pela apropriação do contexto da pessoa com câncer e sua família (no Serviço de Oncologia e domicílio), na qual passamos a fazer parte dele, garantindo maior aproximação com o objeto pesquisado, até o término da coleta de dados.

(c) Encontros informais progredirão para conversas que devem abordar temas cada vez mais complexos, chegando a ter a duração igual ou superior a uma hora - As conversas com as pessoas da família se iniciavam com informalidade, em atividades como ver as fotografias e vídeos das famílias; no decorrer, se aprofundavam pelos fatos do passado e os que estavam acontecendo (com a presença do câncer e do tratamento). Manifestaram-se pela verbalização dos sentimentos, como medos, angústias e esperança, além de expressões de choro e momentos de alegria. Foi realizado um total de cinco entrevistas com as famílias, no domicílio, com duração média de 60 minutos com cada uma delas, somando 15 horas de entrevista, e perfazendo 100 horas de observação, sendo 20 horas no Serviço de Oncologia e 80 horas no contexto domiciliar.

(d) Os processos proximais que se estabelecem nesses encontros servem de base para todo o processo de pesquisa, sendo fundamental a postura de informalidade e conversa, possibilitando o diálogo sobre pontos não diretamente relacionados ao objetivo do estudo - Os encontros no domicílio das famílias permitiram que relatassem as visitas que recebiam do pastor e dos amigos; questões relacionadas às dificuldades que encontravam para transitar nos serviços de saúde, como a demora para conseguir agendar um exame de tomografia; e também questões econômicas, como as dificuldades financeiras referentes ao baixo preço dos produtos agrícolas que produziam.

(e) Os temas abordados nas entrevistas são interessantes e estimulantes para os pesquisadores e para os participantes, pois exploram as histórias de vida e a forma como se dá o desenvolvimento do cuidado no contexto - As famílias rurais, ao falarem sobre suas experiências de vida, tiveram a oportunidade de refletir e aprender com elas. A visualização das fotos e a construção do genograma e do ecomapa proporcionaram reflexões sobre seus vínculos, suas interações e processos de viver e cuidar, ao longo do tempo. Além disso, relatos da vida em família e na comunidade, como a participação em festas religiosas, casamentos e aniversários, permearam todo o processo investigativo.

Dessa forma, o processo proximal intrafamiliar ocorreu por meio do compartilhamento dos mesmos espaços físicos - o domicílio e o Serviço de Oncologia, e desenvolvendo uma mesma atividade - a entrevista. Aconteceu também por meio do estabelecimento da comunicação entre as pessoas, em conversas, e da expressão de sentimentos sobre o câncer, o tratamento e a realização de práticas de cuidado no contexto familiar. A aproximação da família ocorreu, ainda, no momento da elaboração do genograma e do ecomapa, pois houve o resgate de pessoas e momentos históricos da família.

O método de inserção ecológica foi de extrema importância para conhecer as práticas de cuidado dentro das famílias, no cuidar à pessoa com câncer. "Na pesquisa qualitativa, principalmente, pode-se dizer que a interação do pesquisador com os participantes gera uma transferência de energia, produzindo, assim, processos proximais em ambos". ${ }^{9: 521}$

Para compreender como ocorriam as interações entre a família e a pessoa com câncer, na 
realização de práticas de cuidado, foram utilizados os quatro componentes do Modelo Bioecológico, ${ }^{6}$ os quais serão apresentados a seguir.

No primeiro componente - pessoa - foram consideradas algumas características das pessoas das famílias estudadas, como, por exemplo, cor da pele, gênero, etnia, local de origem e religião.

O segundo elemento - processo - foi analisado pela forma como cada pessoa significava suas experiências e interpretava o ambiente. É a interação de pessoas com outras pessoas, com símbolos, objetos e contexto. ${ }^{6}$ Para análise, consideramos a interação da família rural na realização de práticas de cuidado diárias à pessoa com câncer, que promovem o desenvolvimento de todos os envolvidos no cuidado, ou seja, era o que iria mover a família à interação para uma atividade em comum - o cuidar.

No terceiro elemento - o contexto - foram considerados os ambientes em que as pessoas da família rural viviam e transitavam, como o domicílio, a vizinhança, a igreja, a comunidade e os serviços de saúde. Para análise do contexto em que viviam as famílias e a pessoa com câncer, partiu-se para a compreensão do microssistema, que se configura no domicílio das famílias. Ele é descrito em seus aspectos físicos, como também em relação às interações que ocorriam entre os membros da família, como o cuidado à pessoa com câncer e o cuidado entre familiares; pelo modo como os familiares circulavam no domicílio; e nas funções desempenhadas pelas pessoas, em determinadas atividades. $\mathrm{Na}$ análise do mesossistema, foram incluídas as inter-relações entre dois ou mais ambientes onde se encontrava a família rural, como, por exemplo, as interações da pessoa com câncer com outras pessoas, amigos, vizinhos, ou seja, membros da comunidade e dos sistemas de saúde. Ao analisar o exossistema, sendo este o ambiente que não envolve a pessoa com câncer em desenvolvimento como participante ativo, pôde-se constatar, na entrevista com as famílias rurais, que havia influência de pessoas dos serviços de saúde, de farmácias, fornecendo orientações e subsídios aos integrantes das famílias para a realização de práticas de cuidado. No macrossistema, observou-se a influência dos aspectos sociais, econômicos e culturais nas relações familiares. As famílias rurais tinham o apoio da Associação de Apoio a Pessoas com Câncer (AAPECAN). Investigaram-se também os valores, crenças e hábitos da família rural, sobre as práticas de cuidado à pessoa com câncer.
Para análise de um dos contextos da família foram utilizados o ecomapa e o genograma. $\mathrm{O}$ genograma ajudou os membros das famílias a dialogarem sobre suas características e propósitos, unindo as famílias durante sua realização. Além disso, permitiu ao entrevistador a identificação de problemas familiares, ao longo do tempo e do espaço. O ecomapa e o genograma apresentam uma visão geral da família e das relações entre a família e o mundo. Representam a relação da família com os sistemas mais amplos. ${ }^{10}$ Além disso, os referidos instrumentos proporcionaram aos familiares resgatarem pessoas e momentos da história da família. ${ }^{11}$

O último elemento - o tempo - diz respeito à sequência de eventos que constitui a história $\mathrm{e}$ as rotinas de uma pessoa. ${ }^{7}$ Nos estudos de base ecológica, o tempo permite identificar estabilidade ou instabilidade nos ambientes. Ele se reflete no processo de desenvolvimento, uma vez que o ciclo vital é caracterizado por experiências e sucessivas transições nas relações da pessoa com o contexto da interdependência das influências sociais e históricas, que são expressas no curso da vida e nas relações interpessoais. Sendo esse processo permeado pela temporalidade na existência humana, e a sua compreensão permitindo uma visão do desenvolvimento contextualizada e coerente. ${ }^{6}$ Ele desvenda igualmente as raízes históricas das sociedades, as descendências étnicas e a valorização ou não de determinadas práticas culturais ou ritualísticas, organizando também as rotinas e os eventos. ${ }^{12}$ Dessa forma, para analisá-lo junto às famílias da pessoa com câncer, investigamos as práticas de cuidado diárias realizadas através das gerações, o que foi revelado também pelo genograma.

Nenhuma característica pessoal exerce influência de forma isolada, sendo que cada qualidade humana é envolvida e encontra seus significados e profundas expressões em ambientes particulares, tendo a família o principal exemplo. É dentro desses ambientes que a pessoa em desenvolvimento engaja-se e experiencia atividades, papéis, e relações interpessoais, e uma relação direta com fatores físicos, sociais e simbólicos. ${ }^{13}$

As interações ocorrem com pessoas, mas também envolvem símbolos e objetos que se apresentam nos diferentes contextos. ${ }^{6}$ A interação implica em constante troca com os outros e com os ambientes, ou seja, provoca alterações em ambas as partes. A reciprocidade estimula 
e mobiliza as pessoas a se engajarem em uma atividade e a preservarem padrões de interação mais complexos. ${ }^{14}$

Na prática das famílias rurais o cuidado é um veículo promotor para que se estabeleçam os processos proximais, ou seja, as interações entre os membros da família, a pessoa com câncer e outros integrantes dos demais sistemas. "Tanto desenvolvimento quanto saúde resultam de um processo de interação contínua entre a pessoa e o seu contexto" ${ }^{15: 94}$

Assim, os programas de lazer que as famílias rurais realizavam e frequentavam, como festas, casamentos, encontros com vizinhos, amigos, ou com toda a comunidade, poderiam servir como um contexto potencial para promover práticas de cuidado à pessoa com câncer, uma vez que se estabeleciam as interações formando uma rede de apoio social. Era nesses contextos que as famílias rurais transitavam e, na maioria das vezes, poderiam fortalecer ou fragilizar aqueles que estavam envolvidos no processo de cuidar.

\section{CONSIDERAÇÕES FINAIS}

Como contribuição, acreditamos que há caminhos possíveis para cuidar e intervir em famílias que vivenciam situações de adoecer, utilizando metodologias com enfoque sistêmico, que possibilitem analisá-las em diversos ângulos. Destacamos que o presente relato proporcionou gerar conhecimentos relativos à utilização de metodologias, contribuindo para a assistência de enfermagem a famílias.

A Inserção Ecológica demonstrou ser um método adequado para avaliação das interações estabelecidas pela pessoa com câncer, nos contextos de quimioterapia, e com os familiares, no espaço domiciliar, quando eram realizadas as práticas de cuidado. Compreende uma nova maneira de olhar o contexto em que a pessoa está inserida, ou seja, para investigar não apenas características da pessoa, mas também as características do ambiente em que vive.

O Modelo Bioecológico de Urie Bronfenbrenner apresentou-se como alternativa promissora para orientar pesquisas na área da enfermagem, pois, ao se utilizar os quatro núcleos - processo, pessoa, contexto e tempo, isso permite obter uma concepção sistêmica independente do objeto a ser investigado. Entendemos serem necessárias mais investigações voltadas para o contexto das famílias rurais, visando ampliar a assistência à sua saúde.

\section{REFERÊNCIAS}

1. Althoff CR, Elsen I. In: Althoff CR, Elsen I, Nitschke RG, organizadores. Pesquisando a família: olhares contemporâneos. Florianópolis (SC): Papa-livro. 2004. p. 19-28.

2. Schwartz E. O viver o adoecer e o cuidar das famílias de uma comunidade rural do extremo Sul do Brasil: uma perspectiva ecológica [tese]. Florianópolis (SC): Universidade Federal de Santa Catarina, Programa de Pós-Graduação em Enfermagem; 2002.

3. Martins CS. A compreensão de família sob a ótica de pais e filhos envolvidos na violência doméstica contra crianças e adolescentes [dissertação]. Ribeirão Preto (SP): Universidade de São Paulo, Escola de Enfermagem de Ribeirão Preto; 2005.

4. Silva LC. Violência intrafamiliar contra crianças e adolescentes: uma prática educativa com profissionais da educação [dissertação]. Rio Grande (RS): Fundação Universidade Federal do Rio Grande; 2006.

5. Meincke SMK. A construção da paternidade na família do pai adolescente: contribuição para o cuidado de enfermagem [tese]. Florianópolis (SC): Universidade Federal de Santa Catarina, Programa de Pós-Graduação em Enfermagem; 2007.

6. Bronfenbrenner U, Morris PA. The ecology of desenvolmental process. In: Damon W, Sigel IE, Renninger KA, editors. Handbook of child psychology. New York (US): John Wiley \& Sons; 1998. p. 993-1027.

7. Bronfenbrenner U. A ecologia do desenvolvimento: experimentos naturais e planejados. Porto Alegre (RS): Artes Médicas; 1996.

8. Bronfenbrenner U, Ceci SJ. Nature-nurture reconceptualized in desenvolvimental perspective: a bioecological model. Psychol Rev. 1994 Out; 101(4):568-86.

9. Ceconello AM, Koller S. Inserção ecológica na comunidade: uma proposta metodológica para o estudo de famílias em situação de risco. Psicol Reflex Crit. 2003; 16(3):515-24.

10. Wright L, Leahey M. Enfermeiras e famílias: um guia para avaliação e intervenção na família. $4^{\mathrm{a}}$ ed. São Pulo (SP): Roca, 2009.

11. Di Primio AO, Schwartz E, Bielemann VLM, Burille A, Zillmer JGV, Feijó AM. Rede social e vínculos apoiadores das famílias de crianças com câncer. Texto Contexto Enferm. 2010 Abr-Jun; 19(2):334-42.

12. Antoni C, Koller S. A pesquisa ecológica sobre violência no microssistema familiar. In: Koller $S$, organizador. Ecologia do desenvolvimento humano: pesquisa e intervenção no Brasil. Porto Alegre (RS): Casa do Psicólogo; 2004. p. 315-58.

13. Bronfenbrenner U. Ecological system theory. In: Vasta R. organizador. Six theories of child development: 
revised formulations and current issues. London (UK): Jessica Knigsley. 1992. p. 187-249.

14. Prati L, Couto MCPP, Moura A, Poletto M, Koller SH. Revisando a Inserção Ecológica: uma proposta de sistematização. Psicol Reflex Crit. 2008; 21(1):160-9.
15. Morais NA, Koller S. Abordagem ecológica do desenvolvimento humano, psicologia positiva e resiliência: ênfase na saúde. In: Koller S, organizador. Ecologia do desenvolvimento humano: pesquisa e intervenção no Brasil. 2004. p. 96-112. 